\title{
RainNet v1.0: a convolutional neural network for radar-based precipitation nowcasting
}

\author{
Georgy Ayzel $^{1}$, Tobias Scheffer ${ }^{2}$, and Maik Heistermann ${ }^{1}$ \\ ${ }^{1}$ Institute for Environmental Sciences and Geography, University of Potsdam, Potsdam, Germany \\ ${ }^{2}$ Department of Computer Science, University of Potsdam, Potsdam, Germany
}

Correspondence: Georgy Ayzel (ayzel@uni-potsdam.de)

Received: 30 January 2020 - Discussion started: 4 March 2020

Revised: 7 May 2020 - Accepted: 13 May 2020 - Published: 11 June 2020

\begin{abstract}
In this study, we present RainNet, a deep convolutional neural network for radar-based precipitation nowcasting. Its design was inspired by the U-Net and SegNet families of deep learning models, which were originally designed for binary segmentation tasks. RainNet was trained to predict continuous precipitation intensities at a lead time of $5 \mathrm{~min}$, using several years of quality-controlled weather radar composites provided by the German Weather Service (DWD). That data set covers Germany with a spatial domain of $900 \mathrm{~km} \times 900 \mathrm{~km}$ and has a resolution of $1 \mathrm{~km}$ in space and $5 \mathrm{~min}$ in time. Independent verification experiments were carried out on 11 summer precipitation events from 2016 to 2017. In order to achieve a lead time of $1 \mathrm{~h}$, a recursive approach was implemented by using RainNet predictions at $5 \mathrm{~min}$ lead times as model inputs for longer lead times. In the verification experiments, trivial Eulerian persistence and a conventional model based on optical flow served as benchmarks. The latter is available in the rainymotion library and had previously been shown to outperform DWD's operational nowcasting model for the same set of verification events.

RainNet significantly outperforms the benchmark models at all lead times up to $60 \mathrm{~min}$ for the routine verification metrics mean absolute error (MAE) and the critical success in$\operatorname{dex}(\mathrm{CSI})$ at intensity thresholds of $0.125,1$, and $5 \mathrm{~mm} \mathrm{~h}^{-1}$. However, rainymotion turned out to be superior in predicting the exceedance of higher intensity thresholds (here 10 and $15 \mathrm{~mm} \mathrm{~h}^{-1}$ ). The limited ability of RainNet to predict heavy rainfall intensities is an undesirable property which we attribute to a high level of spatial smoothing introduced by the model. At a lead time of $5 \mathrm{~min}$, an analysis of power spectral density confirmed a significant loss of spectral power at
\end{abstract}

length scales of $16 \mathrm{~km}$ and below. Obviously, RainNet had learned an optimal level of smoothing to produce a nowcast at 5 min lead time. In that sense, the loss of spectral power at small scales is informative, too, as it reflects the limits of predictability as a function of spatial scale. Beyond the lead time of $5 \mathrm{~min}$, however, the increasing level of smoothing is a mere artifact - an analogue to numerical diffusion - that is not a property of RainNet itself but of its recursive application. In the context of early warning, the smoothing is particularly unfavorable since pronounced features of intense precipitation tend to get lost over longer lead times. Hence, we propose several options to address this issue in prospective research, including an adjustment of the loss function for model training, model training for longer lead times, and the prediction of threshold exceedance in terms of a binary segmentation task. Furthermore, we suggest additional input data that could help to better identify situations with imminent precipitation dynamics. The model code, pretrained weights, and training data are provided in open repositories as an input for such future studies.

\section{Introduction}

The term "nowcasting" refers to forecasts of precipitation field movement and evolution at high spatiotemporal resolutions (1-10 min, 100-1000 m) and short lead times (minutes to a few hours). Nowcasts have become popular not only with a broad civil community for planning everyday activities; they are particularly relevant as part of early warning systems for heavy rainfall and related impacts such as flash floods or landslides. While the recent advances in high- 
performance computing and data assimilation significantly improved numerical weather prediction (NWP) (Bauer et al., 2015), the computational resources required to forecast precipitation field dynamics at very high spatial and temporal resolutions are typically prohibitive for the frequent update cycles $(5-10 \mathrm{~min})$ that are required for operational nowcasting systems. Furthermore, the heuristic extrapolation of precipitation dynamics that are observed by weather radars still outperforms NWP forecasts at short lead times (Lin et al., 2005; Sun et al., 2014). Thus, the development of new nowcasting systems based on parsimonious but reliable and fast techniques remains an essential trait in both atmospheric and natural hazard research.

There are many nowcasting systems which work operationally all around the world to provide precipitation nowcasts (Reyniers, 2008; Wilson et al., 1998). These systems, at their core, utilize a two-step procedure that was originally suggested by Austin and Bellon (1974), consisting of tracking and extrapolation. In the tracking step, a velocity is obtained from a series of consecutive radar images. In the extrapolation step, that velocity is used to propagate the most recent precipitation observation into the future. Various flavors and variations of this fundamental idea have been developed and operationalized over the past decades, which provide value to users of corresponding products. Still, the fundamental approach to nowcasting has not changed much over recent years - a situation that might change with the increasing popularity of deep learning in various scientific disciplines.

"Deep learning" refers to machine-learning methods for artificial neural networks with "deep" architectures. Rather than relying on engineered features, deep learning derives low-level image features on the lowest layers of a hierarchical network and increasingly abstract features on the highlevel network layers as part of the solution of an optimization problem based on training data (LeCun et al., 2015). Deep learning began its rise from the field of computer science when it started to dramatically outperform reference methods in image classification (Krizhevsky et al., 2012) and machine translation (Sutskever et al., 2014), which was followed by speech recognition (LeCun et al., 2015). Three main reasons caused this substantial breakthrough in predictive efficacy: the availability of "big data" for model training, the development of activation functions and network architectures that result in numerically stable gradients across many network layers (Dahl et al., 2013), and the ability to scale the learning process massively through parallelization on graphics processing units (GPUs). Today, deep learning is rapidly spreading into many data-rich scientific disciplines, and it complements researchers' toolboxes with efficient predictive models, including in the field of geosciences (Reichstein et al., 2019).

While expectations in the atmospheric sciences are high (see, e.g., Dueben and Bauer, 2018; Gentine et al., 2018), the investigation of deep learning in radar-based precipita- tion nowcasting is still in its infancy, and universal solutions are not yet available. Shi et al. (2015) were the first to introduce deep learning models in the field of radar-based precipitation nowcasting: they presented a convolutional long short-term memory (ConvLSTM) architecture, which outperformed the optical-flow-based ROVER (Real-time Optical flow by Variational methods for Echoes of Radar) nowcasting system in the Hong Kong area. A follow-up study (Shi et al., 2017) introduced new deep learning architectures, namely the trajectory gated recurrent unit (TrajGRU) and the convolutional gated recurrent unit (ConvGRU), and demonstrated that these models outperform the ROVER nowcasting system, too. Further studies by Singh et al. (2017) and Shi et al. (2018) confirmed the potential of deep learning models for radar-based precipitation nowcasting for different sites in the US and China. Most recently, Agrawal et al. (2019) introduced a U-Net-based deep learning model for the prediction of the exceedance of specific rainfall intensity thresholds compared to optical flow and numerical weather prediction models. Hence, the exploration of deep learning techniques in radar-based nowcasting has begun, and the potential to overcome the limitations of standard tracking and extrapolation techniques has become apparent. There is a strong need, though, to further investigate different architectures, to set up new benchmark experiments, and to understand under which conditions deep learning models can be a viable option for operational services.

In this paper, we introduce RainNet - a deep neural network which aims at learning representations of spatiotemporal precipitation field movement and evolution from a massive, open radar data archive to provide skillful precipitation nowcasts. The present study outlines RainNet's architecture and its training and reports on a set of benchmark experiments in which RainNet competes against a conventional nowcasting model based on optical flow. Based on these experiments, we evaluate the potential of RainNet for nowcasting but also its limitations in comparison to conventional radar-based nowcasting techniques. Based on this evaluation, we attempt to highlight options for future research towards the application of deep learning in the field of precipitation nowcasting.

\section{Model description}

\subsection{Network architecture}

To investigate the potential of deep neural networks for radarbased precipitation nowcasting, we developed RainNet a convolutional deep neural network (Fig. 1). Its architecture was inspired by the U-Net and SegNet families of deep learning models for binary segmentation (Badrinarayanan et al., 2017; Ronneberger et al., 2015; Iglovikov and Shvets, 2018). These models follow an encoder-decoder architecture in which the encoder progressively downscales the spatial 


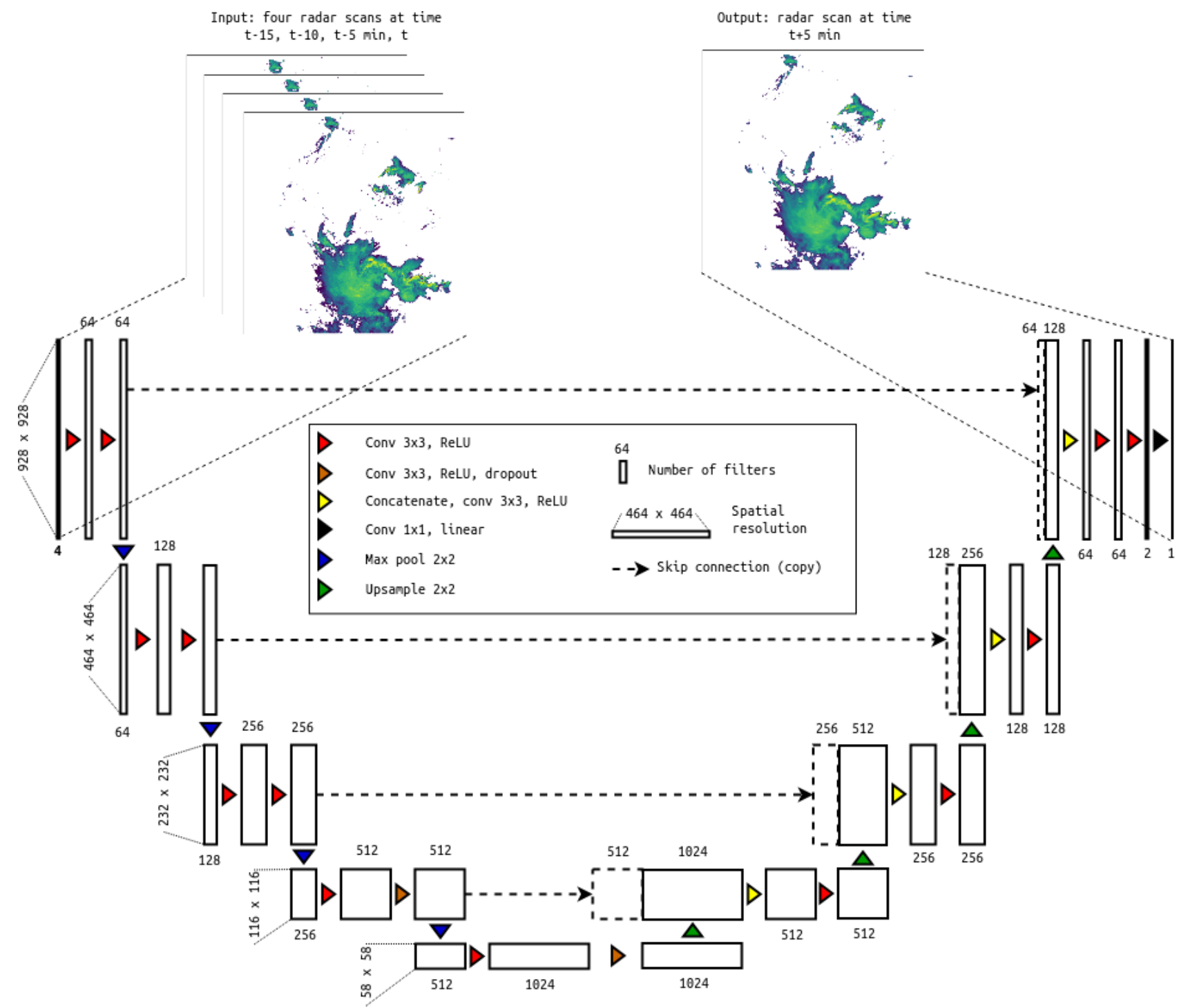

Figure 1. Illustration of the RainNet architecture. RainNet is a convolutional deep neural network which follows a standard encoder-decoder structure with skip connections between its branches. See main text for further explanation.

resolution using pooling, followed by convolutional layers, and the decoder progressively upscales the learned patterns to a higher spatial resolution using upsampling, followed by convolutional layers. There are skip connections (Srivastava et al., 2015) from the encoder to the decoder in order to ensure semantic connectivity between features on different layers.

As elementary building blocks, RainNet has 20 convolutional, 4 max pooling, 4 upsampling, and 2 dropout layers and 4 skip connections. Convolutional layers aim to generate data-driven spatial features from the corresponding input volume using several convolutional filters. Each filter is a three-dimensional tensor of learnable weights with a small spatial kernel size (e.g., $3 \times 3$, and the third dimension equal to that of the input volume). A filter convolves through the in- put volume with a step-size parameter (or stride; stride $=1$ in this study) and produces a dot product between filter weights and corresponding input volume values. A bias parameter is added to this dot product, and the results are transformed using an adequate activation function. The purpose of the activation function is to add nonlinearities to the convolutional layer output - to enrich it to learn nonlinear features. To increase the efficiency of convolutional layers, it is necessary to optimize their hyperparameters (such as number of filters, kernel size, and type of activation function). This has been done in a heuristic tuning procedure (not shown). As a result, we use convolutional layers with up to 1024 filters, kernel sizes of $1 \times 1$ and $3 \times 3$, and linear or rectified linear unit (ReLU; Nair and Hinton, 2010) activation functions. 
Using a max pooling layer has two primary reasons: it achieves an invariance to scale transformations of detected features and increases the network's robustness to noise and clutter (Boureau et al., 2010). The filter of a max pooling layer slides over the input volume independently for every feature map with some step parameter (or stride) and resizes it spatially using the maximum (max) operator. In our study, each max pooling layer filter is $2 \times 2$ in size, applied with a stride of 2 . Thus, we take the maximum of four numbers in the filter region $(2 \times 2)$, which downsamples our input volume by a factor of 2 . In contrast to a max pooling layer, an upsampling layer is designed for the spatial upsampling of the input volume (Long et al., 2015). An upsampling layer operator slides over the input volume and fills (copies) each input value to a region that is defined by the upsampling kernel size $(2 \times 2$ in this study).

Skip connections were proposed by Srivastava et al. (2015) in order to avoid the problem of vanishing gradients for the training of very deep neural networks. Today, skip connections are a standard group of methods for any form of information transfer between different layers in a neural network (Gu et al., 2018). They allow for the most common patterns learned on the bottom layers to be reused by the top layers in order to maintain a connection between different data representations along the whole network. Skip connections turned out to be crucial for deep neural network efficiency in recent studies (Iglovikov and Shvets, 2018). For RainNet, we use skip connections for the transition of learned patterns from the encoder to the decoder branch at the different resolution levels.

One of the prerequisites for U-Net-based architectures is that the spatial extent of input data has to be a multiple of $2^{n+1}$, where $n$ is the number of max pooling layers. As a consequence, the spatial extent on different resolution levels becomes identical for the decoder and encoder branches. Correspondingly, the radar composite grids were transformed from the native spatial extent of 900 cells $\times 900$ cells to the extent of 928 cells $\times 928$ cells using mirror padding.

RainNet takes four consecutive radar composite grids as separate input channels $(t-15, t-10$, and $t-5 \mathrm{~min}$ and $t$, where $t$ is the time of the nowcast) to produce a nowcast at time $t+5$ min. Each grid contains 928 cells $\times 928$ cells with an edge length of $1 \mathrm{~km}$; for each cell, the input value is the logarithmic precipitation depth as retrieved from the radarbased precipitation product. There are five almost symmetrical resolution levels for both decoder and encoder which utilize precipitation patterns at the full spatial input resolution of $(x, y)$, at half resolution $(x / 2, y / 2)$, at $(x / 4, y / 4)$, at $(x / 8, y / 8)$, and at $(x / 16, y / 16)$. To increase the robustness and to prevent the overfitting of pattern representations at coarse resolutions, we implemented a dropout regularization technique (Srivastava et al., 2014). Finally, the output layer of resolution $(x, y)$ with a linear activation function provides the predicted logarithmic precipitation (in millimeters) in each grid cell for $t+5 \mathrm{~min}$.
RainNet differs fundamentally from ConvLSTM (Shi et al., 2015), a prior neural-network approach, which accounts for both spatial and temporal structures in radar data by using stacked convolutional and long short-term memory (LSTM) layers that preserve the spatial resolution of the input data alongside all the computational layers. LSTM networks have been observed to be brittle; in several application domains, convolutional neural networks have turned out to be numerically more stable during training and make more accurate predictions than these recurrent neural networks (e.g., Bai et al., 2018; Gehring et al., 2017).

Therefore, RainNet uses a fully convolutional architecture and does not use LSTM layers to propagate information through time. In order to make predictions with a larger lead time, we apply RainNet recursively. After predicting the estimated $\log$ precipitation for $t+5 \mathrm{~min}$, the measured values for $t-10, t-5$, and $t$, as well as the estimated value for $t+5$, serve as the next input volume which yields the estimated log precipitation for $t+10 \mathrm{~min}$. The input window is then moved on incrementally.

\subsection{Optimization procedure}

In total, RainNet has almost 31.4 million parameters. We optimized these parameters using a procedure of which we show one iteration in Fig. 2: first, we read a sample of input data that consists of radar composite grids at time $t-15$, $t-10$, and $t-5 \mathrm{~min}$ and $t$, as well as a sample of the observed precipitation at time $t+5$. For both input and observation, we increase the spatial extent to $928 \times 928$ using mirror padding and transform precipitation depth $x\left(\mathrm{~mm} 5 \mathrm{~min}^{-1}\right)$ as follows (Eq. 1):

$x_{\text {transformed }}=\ln \left(x_{\text {raw }}+0.01\right)$.

Second, RainNet carries out a prediction based on the input data. Third, we calculate a loss function that represents the deviation between prediction and observation. Previously, Chen et al. (2018) showed that using the logcosh loss function is beneficial for the optimization of variational autoencoders (VAEs) in comparison to mean squared error. Accordingly, we employed the logcosh loss function as follows (Eq. 2):

$$
\begin{aligned}
& \text { Loss }=\frac{\sum_{i=1}^{n} \ln \left(\cosh \left(\operatorname{now}_{i}-\mathrm{obs}_{i}\right)\right)}{n}, \\
& \cosh (x)=\frac{1}{2}\left(e^{x}+e^{-x}\right),
\end{aligned}
$$

where now $_{i}$ and obs $i$ are nowcast and observation at the $i$ th location, respectively, cosh is the hyperbolic cosine function (Eq. 3), and $n$ is the number of cells in the radar composite grid.

Fourth, we update RainNet's model parameters to minimize the loss function using a backpropagation algorithm where the Adam optimizer is utilized to compute the gradients (Kingma and $\mathrm{Ba}, 2015$ ). 


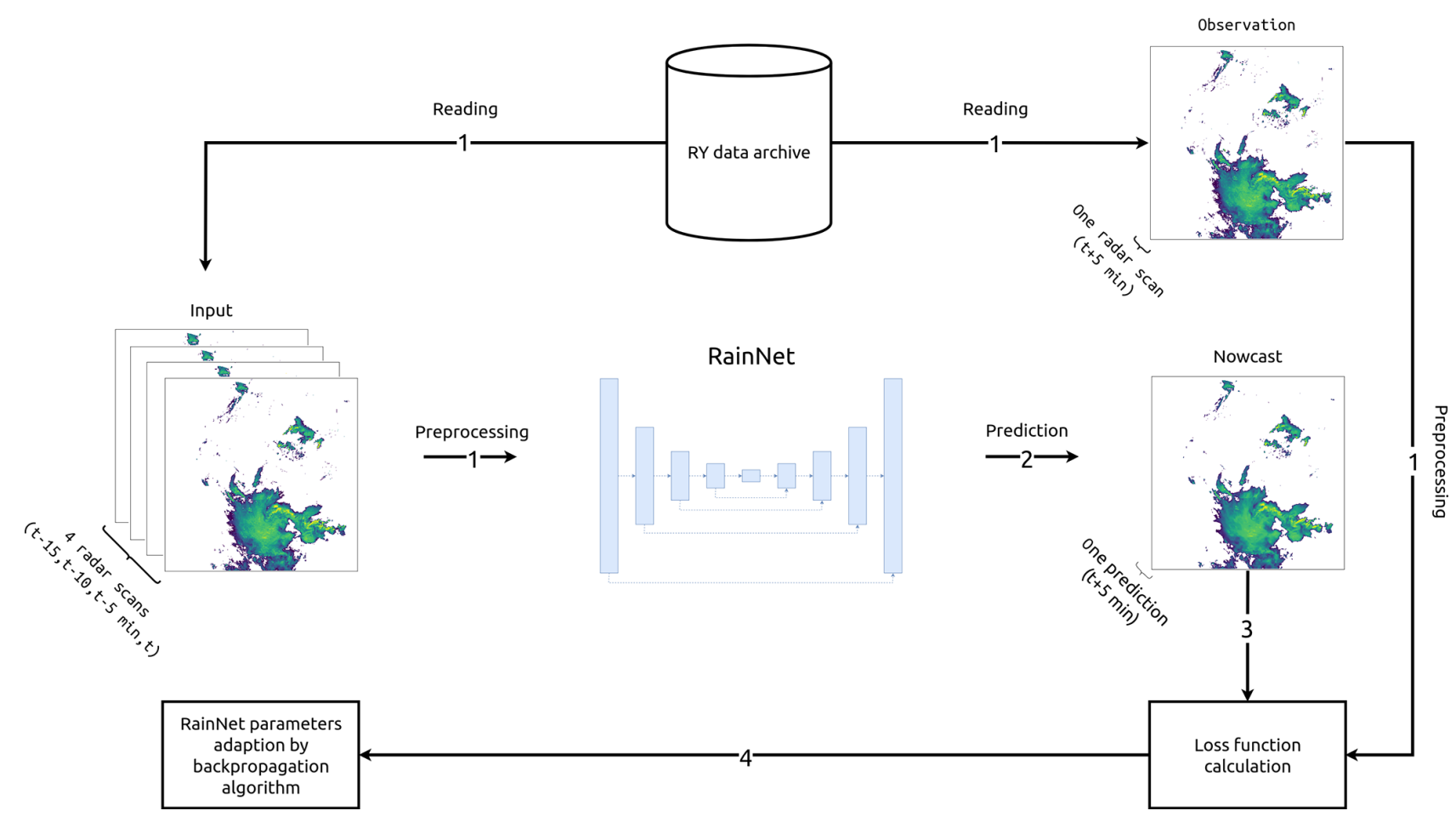

Figure 2. Illustration of one iteration step of the RainNet parameters optimization procedure.

We optimized RainNet's parameters using 10 epochs (one epoch ends when the neural network has seen every input data sample once; then the next epoch begins) with a mini batch of size 2 (one mini batch holds a few input data samples). The optimization procedure converged on the eighth epoch, showing the saturation of RainNet's performance on the validation data. The learning rate of the Adam optimizer had a value of $1 \times 10^{-4}$, while other parameters had default values from the original paper of Kingma and $\mathrm{Ba}$ (2015).

The entire setup was empirically identified as the most successful in terms of RainNet's performance on validation data, while other configurations with different loss functions (e.g., mean absolute error, mean squared error) and optimization algorithms (e.g., stochastic gradient descent) also converged. The average training time on a single GPU (NVIDIA GeForce GTX 1080Ti, NVIDIA GTX TITAN X, or NVIDIA Tesla P100) varies from 72 to $76 \mathrm{~h}$.

We support this paper by a corresponding repository on GitHub (https://github.com/hydrogo/rainnet; last access: 10 June 2020; Ayzel, 2020a), which holds the RainNet model architecture written in the Python 3 programming language (https://python.org, last access: 28 January 2020) using the Keras deep learning library (Chollet et al., 2015) alongside its parameters (Ayzel, 2020b), which had been optimized on the radar data set described in the following section.

\section{Data and experimental setup}

\subsection{Radar data}

We use the RY product of the German Weather Service (DWD) as input data for training and validating the RainNet model. The RY product represents a quality-controlled rainfall-depth composite of 17 operational DWD Doppler radars. It has a spatial extent of $900 \mathrm{~km} \times 900 \mathrm{~km}$, covers the whole area of Germany, and has been available since 2006 . The spatial and temporal resolution of the RY product is $1 \mathrm{~km} \times 1 \mathrm{~km}$ and $5 \mathrm{~min}$, respectively.

In this study, we use RY data that cover the period from 2006 to 2017. We split the available RY data as follows: while we use data from 2006 to 2013 to optimize RainNet's model parameters and data from 2014 to 2015 to validate RainNet's performance, data from 2016 to 2017 are used for model verification (Sect. 3.3). For both optimization and validation periods, we keep only data from May to September and ignore time steps for which the precipitation field (with rainfall intensity more than $0.125 \mathrm{~mm} \mathrm{~h}^{-1}$ ) covers less than $10 \%$ of the RY domain. For each subset of the data - for optimization, validation, and verification - every time step (or frame) is used once as $t_{0}$ (forecast time) so that the resulting sequences that are used as input to a single forecast $\left(t_{0}-15 \mathrm{~min}, \ldots, t_{0}\right)$ overlap in time. The number of resulting sequences amounts to 41988 for the optimization, 5722 for the validation, and 9626 for the verification (see also Sect. 3.3). 


\subsection{Reference models}

We use nowcasting models from the rainymotion Python library (Ayzel et al., 2019) as benchmarks with which we evaluate RainNet. As the first baseline model, we use Eulerian persistence (hereafter referred to as Persistence), which assumes that for any lead time $n(\min )$ precipitation at $t+n$ is the same as at forecast time $t$. Despite its simplicity, it is quite a powerful model for very short lead times, which also establishes a solid verification efficiency baseline which can be achieved with a trivial model without any explicit assumptions. As the second baseline model, we use the Dense model from the rainymotion library (hereafter referred to as Rainymotion), which is based on optical flow techniques for precipitation field tracking and the constant-vector advection scheme for precipitation field extrapolation. Ayzel et al. (2019) showed that this model has an equivalent or even superior performance in comparison to the operational RADVOR (radar real-time forecasting) model from DWD for a wide range of rainfall events.

\subsection{Verification experiments and performance evaluation}

For benchmarking RainNet's predictive skill in comparison to the baseline models, Rainymotion and Persistence, we selected 11 events during the summer months of the verification period (2016-2017). These events were selected for covering a range of event characteristics with different rainfall intensity, spatial coverage, and duration. A detailed account of the events' properties is given by Ayzel et al. (2019).

We use three metrics for model verification: mean absolute error (MAE), critical success index (CSI), and fractions skill score (FSS). Each metric represents a different category of scores. MAE (Eq. 4) corresponds to the continuous category and maps the differences between nowcast and observed rainfall intensities. CSI (Eq. 5) is a categorical score based on a standard contingency table for calculating matches between Boolean variables which indicate the exceedance of specific rainfall intensity thresholds. FSS (Eq. 6) represents neighborhood verification scores and is based on comparing nowcast and observed fractional coverages of rainfall intensities exceeding specific thresholds in spatial neighborhoods (windows) of certain sizes.

$$
\begin{aligned}
& \mathrm{MAE}=\frac{\sum_{i=1}^{n}\left|\mathrm{now}_{i}-\mathrm{obs}_{i}\right|}{n}, \\
& \mathrm{CSI}=\frac{\text { hits }}{\text { hits }+ \text { false alarms }+ \text { misses }}, \\
& \mathrm{FSS}=1-\frac{\sum_{i=1}^{n}\left(P_{\mathrm{n}}-P_{\mathrm{o}}\right)^{2}}{\sum_{i=1}^{n} P_{\mathrm{n}}^{2}+\sum_{i=1}^{n} P_{\mathrm{o}}^{2}},
\end{aligned}
$$

where quantities now $i$ and $\operatorname{obs}_{i}$ are nowcast and observed rainfall rate in the $i$ th pixel of the corresponding radar image, and $n$ is the number of pixels. Hits, false alarms, and misses are defined by the contingency table and the corresponding threshold value. Quantities $P_{\mathrm{n}}$ and $P_{\mathrm{o}}$ represent the nowcast and observed fractions, respectively, of rainfall intensities exceeding a specific threshold for a defined neighborhood size. MAE is positive and unbounded with a perfect score of 0 ; both CSI and FSS can vary from 0 to 1 with a perfect score of 1 . We have applied threshold rain rates of $0.125,1,5,10$, and $15 \mathrm{~mm} \mathrm{~h}^{-1}$ for calculating the CSI and the FSS. For calculating the FSS, we use neighborhood (window) sizes of 1, 5,10 , and $20 \mathrm{~km}$.

The verification metrics we use in this study quantify the models' performance from different perspectives. The MAE captures errors in rainfall rate prediction (the fewer the better), and CSI (the higher the better) captures model accuracy - the fraction of the forecast event that was correctly predicted - but does not distinguish between the sources of errors. The FSS determines how the nowcast skill depends on both the threshold of rainfall exceedance and the spatial scale (Mittermaier and Roberts, 2010).

In addition to standard verification metrics described above, we calculate the power spectral density (PSD) of nowcasts and corresponding observations using Welch's method (Welch, 1967) to investigate the effects of smoothing demonstrated by different models.

\section{Results and discussion}

For each event, RainNet was used to compute nowcasts at lead times from 5 to $60 \mathrm{~min}$ (in $5 \mathrm{~min}$ steps). To predict the precipitation at time $t+5 \mathrm{~min}$ ( $t$ being the forecast time), we used the four latest radar images (at time $t-15, t-10$, and $t-5 \mathrm{~min}$ and $t$ ) as input. Since RainNet was only trained to predict precipitation at $5 \mathrm{~min}$ lead times, predictions beyond $t+5$ were made recursively: in order to predict precipitation at $t+10$, we considered the prediction at $t+5$ as the latest observation. That recursive procedure was repeated up to a maximum lead time of $60 \mathrm{~min}$. Rainymotion uses the two latest radar composite grids $(t-5, t)$ in order to retrieve a velocity field and then to advect the latest radar-based precipitation observation at forecast time $t$ to $t+5, t+10, \ldots$, and $t+60$.

Figure 3 shows the routine verification metrics MAE and CSI for RainNet, Rainymotion, and Persistence as a function of lead time. The preliminary analysis had shown the same general pattern of model efficiency for each of the 11 events (Sect. S1 in the Supplement), which is why we only show the average metrics over all events. The results basically fall into two groups.

The first group includes the MAE and the CSI metrics up to a threshold of $5 \mathrm{~mm} \mathrm{~h}^{-1}$. For these, RainNet clearly outperforms the benchmarks at any lead time (differences between models were tested to be significant with the twotailed $t$ test at a significance level of $5 \%$; results not shown). Persistence is the least skillful, as could be expected for a trivial baseline. The relative differences between RainNet 
and Rainymotion are more pronounced for the MAE than for the CSI. For the MAE, the advance of RainNet over Rainymotion increases with lead time. For the CSI, the superiority of RainNet over Rainymotion appears to be highest for intermediate lead times between 20 and $40 \mathrm{~min}$. The performance of all models, in terms of CSI, decreases with increasing intensity thresholds.

That trend - a decreasing CSI with increasing intensity - continues with the second group of metrics: the CSI for thresholds of 10 and $15 \mathrm{~mm} \mathrm{~h}^{-1}$. For both metrics and any of the competing methods at any lead time, the CSI does not exceed a value of 0.31 (obtained by RainNet at $5 \mathrm{~min}$ lead time and a threshold of $10 \mathrm{~mm} \mathrm{~h}^{-1}$ ). That is below a value of $1 / e \approx 0.37$ which had been suggested by Germann and Zawadzki (2002) as a "limit of predictability" (under the assumption that the optimal value of the metric is 1 and that it follows an exponential-like decay over lead time). Irrespective of such an - admittedly arbitrary - predictability threshold, the loss of skill from an intensity threshold of 5 to $10 \mathrm{~mm} \mathrm{~h}^{-1}$ is remarkable for all competing models. Visually more apparent, however, is another property of the second group of metrics, which is that Rainymotion outperforms RainNet (except for a threshold of $10 \mathrm{~mm} \mathrm{~h}^{-1}$ at lead times of 5 and $60 \mathrm{~min}$ ). That becomes most pronounced for the CSI at $15 \mathrm{~mm} \mathrm{~h}^{-1}$, while RainNet has a similar CSI value as Rainymotion at a lead time of $5 \mathrm{~min}$, it entirely fails at predicting the exceedance of $15 \mathrm{~mm} \mathrm{~h}^{-1}$ for longer lead times.

In summary, Fig. 3 suggests that RainNet outperforms Rainymotion (as a representative of standard tracking and extrapolation techniques based on optical flow) for low and intermediate rain rates (up to $5 \mathrm{~mm} \mathrm{~h}^{-1}$ ). Neither RainNet nor Rainymotion appears to have much skill at predicting the exceedance of $10 \mathrm{~mm} \mathrm{~h}^{-1}$, but the loss of skill for high intensities is particularly remarkable for RainNet, which obviously has difficulties in predicting pronounced precipitation features with high intensities.

In order to better understand the fundamental properties of RainNet predictions in contrast to Rainymotion, we continue by inspecting a nowcast at three different lead times $(5,30$, and $60 \mathrm{~min}$ ) for a verification event at an arbitrarily selected forecast time (29 May 2016, 19:15:00 UTC). The top row of Fig. 4 shows the observed precipitation, and the second and third rows show Rainymotion and RainNet predictions. Since it is visually challenging to track the motion pattern at the scale of $900 \mathrm{~km} \times 900 \mathrm{~km}$ by eye, we illustrate the velocity field as obtained from optical flow, which forms the basis for Rainymotion's prediction. While it is certainly difficult to infer the predictive performance of the two models from this figure, another feature becomes immediately striking: RainNet introduces a spatial smoothing which appears to substantially increase with lead time. In order to quantify that visual impression, we calculated, for the same example, the power spectral density (PSD) of the nowcasts and the corresponding observations (bottom row in Fig. 4), using Welch's method (Welch, 1967). In simple terms, the PSD represents the prominence of precipitation features at different spatial scales, expressed as the spectral power at different wavelengths after a two-dimensional fast Fourier transform. The power spectrum itself is not of specific interest here; it is the loss of power at different length scales, relative to the observation, that is relevant in this context. The loss of power of Rainymotion nowcasts appears to be constrained to spatial scales below $4 \mathrm{~km}$ and does not seem to depend on lead time (see also Ayzel et al., 2019). For RainNet, however, a substantial loss of power at length scales below $16 \mathrm{~km}$ becomes apparent at a lead time of $5 \mathrm{~min}$. For longer lead times of 30 and $60 \mathrm{~min}$, that loss of power grows and propagates to scales of up to $32 \mathrm{~km}$. That loss of power over a range of scales corresponds to our visual impression of spatial smoothing.

In order to investigate whether that loss of spectral power at smaller scales is a general property of RainNet predictions, we computed the PSD for each forecast time in each verification event in order to obtain an average PSD for observations and nowcasts at lead times of 5, 30, and $60 \mathrm{~min}$. The corresponding results are shown in Fig. 5. They confirm that the behavior observed in the bottom row of Fig. 4 is, in fact, representative of the entirety of verification events. Precipitation fields predicted by RainNet are much smoother than both the observed fields and the Rainymotion nowcasts. At a lead time of $5 \mathrm{~min}$, RainNet starts to lose power at a scale of $16 \mathrm{~km}$. That loss accumulates over lead time and becomes effective up to a scale of $32 \mathrm{~km}$ at a lead time of $60 \mathrm{~min}$. These results confirm qualitative findings of Shi et al. (2015, 2018), who described their nowcasts as "smooth" or "fuzzy".

RainNet obviously learned, as the optimal way to minimize the loss function, to introduce a certain level of smoothing for the prediction at time $t+5 \mathrm{~min}$. It might even have learned to systematically "attenuate" high intensity features as a strategy to minimize the loss function, which would be consistent with the results of the CSI at a threshold of $15 \mathrm{~mm} \mathrm{~h}^{-1}$, as shown in Fig. 3. For the sake of simplicity, though, we will refer to the overall effect as "smoothing" in the rest of the paper. According to the loss of spectral power, the smoothing is still small at a length scale of $16 \mathrm{~km}$ but becomes increasingly effective at smaller scales from 2 to $8 \mathrm{~km}$. It is important to note that the loss of power below length scales of $16 \mathrm{~km}$ at a lead time of $5 \mathrm{~min}$ is an essential property of RainNet. It reflects the learning outcome and illustrates how RainNet factors in predictive uncertainty at $5 \mathrm{~min}$ lead times by smoothing over small spatial scales. Conversely, the increasing loss of power and its propagation to larger scales up to $32 \mathrm{~km}$ are not an inherent property of RainNet but a consequence of its recursive application in our study context: as the predictions at short lead times serve as model inputs for predictions at longer lead times, the results become increasingly smooth. So while the smoothing introduced at 5 min lead times can be interpreted as a direct result of the learning procedure, the cumulative smoothing at longer lead times has to rather be considered an artifact simi- 

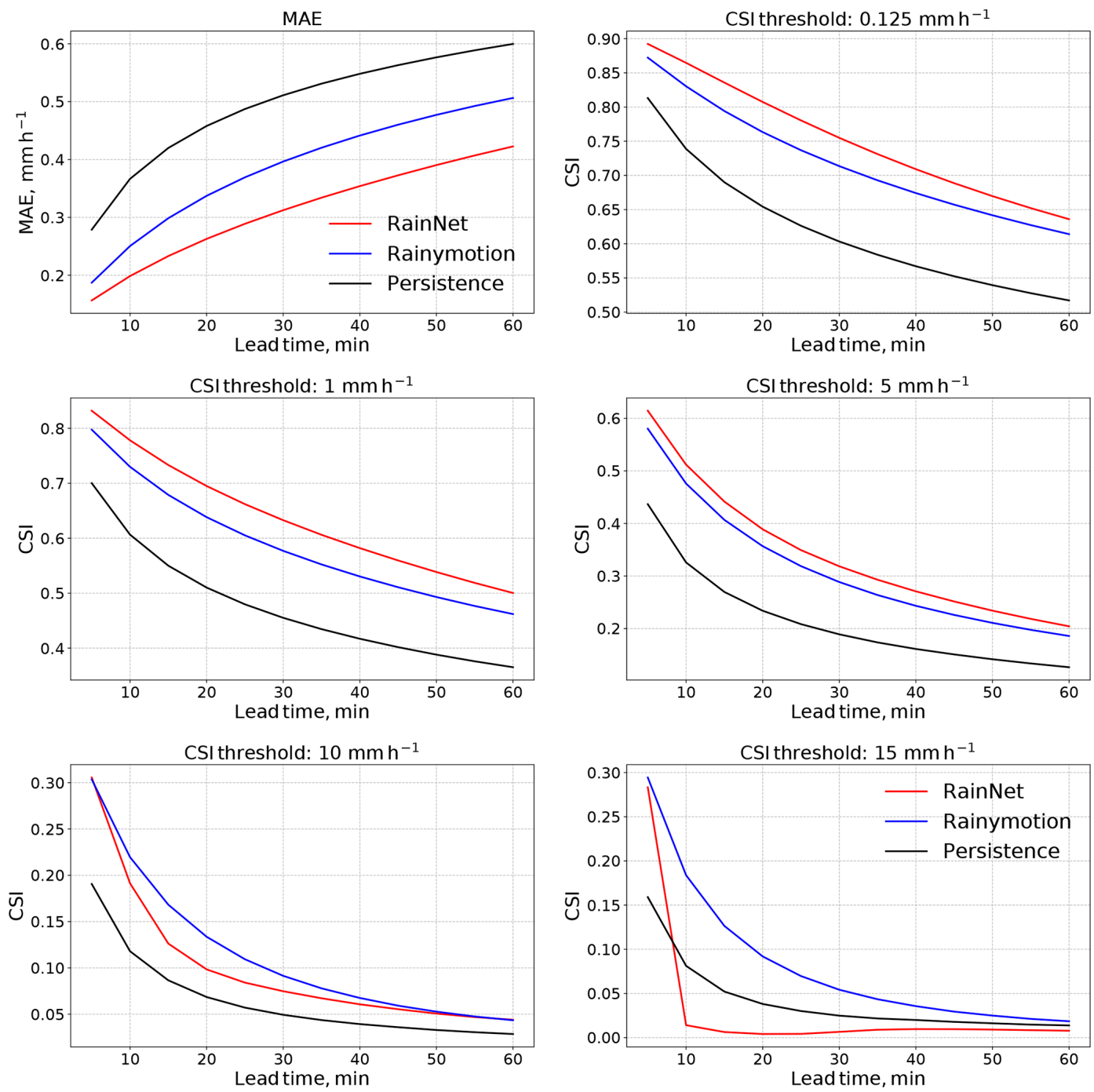

Figure 3. Mean absolute error (MAE) and critical success index (CSI) for five different intensity thresholds $\left(0.125,1,5,10\right.$, and $\left.15 \mathrm{mmh}^{-1}\right)$. The metrics are shown as a function of lead time. All values represent the average of the corresponding metric over all 11 verification events.

lar to the effect of "numerical diffusion" in numerically solving the advection equation.

Given this understanding of RainNet's properties, we used the fractions skill score (FSS) to provide further insight into the dependency of predictive skill on the spatial scale. To that end, the FSS was obtained by comparing the predicted and observed fractional coverage of pixels (inside a spatial window/neighborhood) that exceed a certain intensity threshold (see Eq. 6 in Sect. 3.3). Figure 6 shows the FSS for Rainymotion and RainNet as an average over all verification events, for spatial window sizes of $1,5,10$, and $20 \mathrm{~km}$, and for intensity thresholds of $0.125,1,5,10$, and $15 \mathrm{~mm} \mathrm{~h}^{-1}$. In addition to the color code, the value of the FSS is given for each combination of window size (scale) and intensity. In the case that one model is superior to the other, the correspondingly higher FSS value is highlighted in bold black digits.
Based on the above results and discussion of RainNet's versus Rainymotion's predictive properties, the FSS figures are plausible and provide a more formalized approach to express different behaviors of RainNet and Rainymotion in terms of predictive skill. In general, the skill of both models decreases with decreasing window sizes, increasing lead times, and increasing intensity thresholds. RainNet tends to outperform Rainymotion at lower rainfall intensities (up to $5 \mathrm{~mm} \mathrm{~h}^{-1}$ ) at the native grid resolution (i.e., a window size of $1 \mathrm{~km}$ ). With increasing window sizes and intensity thresholds, Rainymotion becomes the superior model. At an intensity threshold of $5 \mathrm{mmh}^{-1}$, Rainymotion outperforms RainNet at window sizes equal to or greater than $5 \mathrm{~km}$. At intensity thresholds of 10 and $15 \mathrm{~mm} \mathrm{~h}^{-1}$, Rainymotion is superior at any lead time and window size (except a window size of $1 \mathrm{~km}$ for a threshold of $10 \mathrm{~mm} \mathrm{~h}^{-1}$ ). 

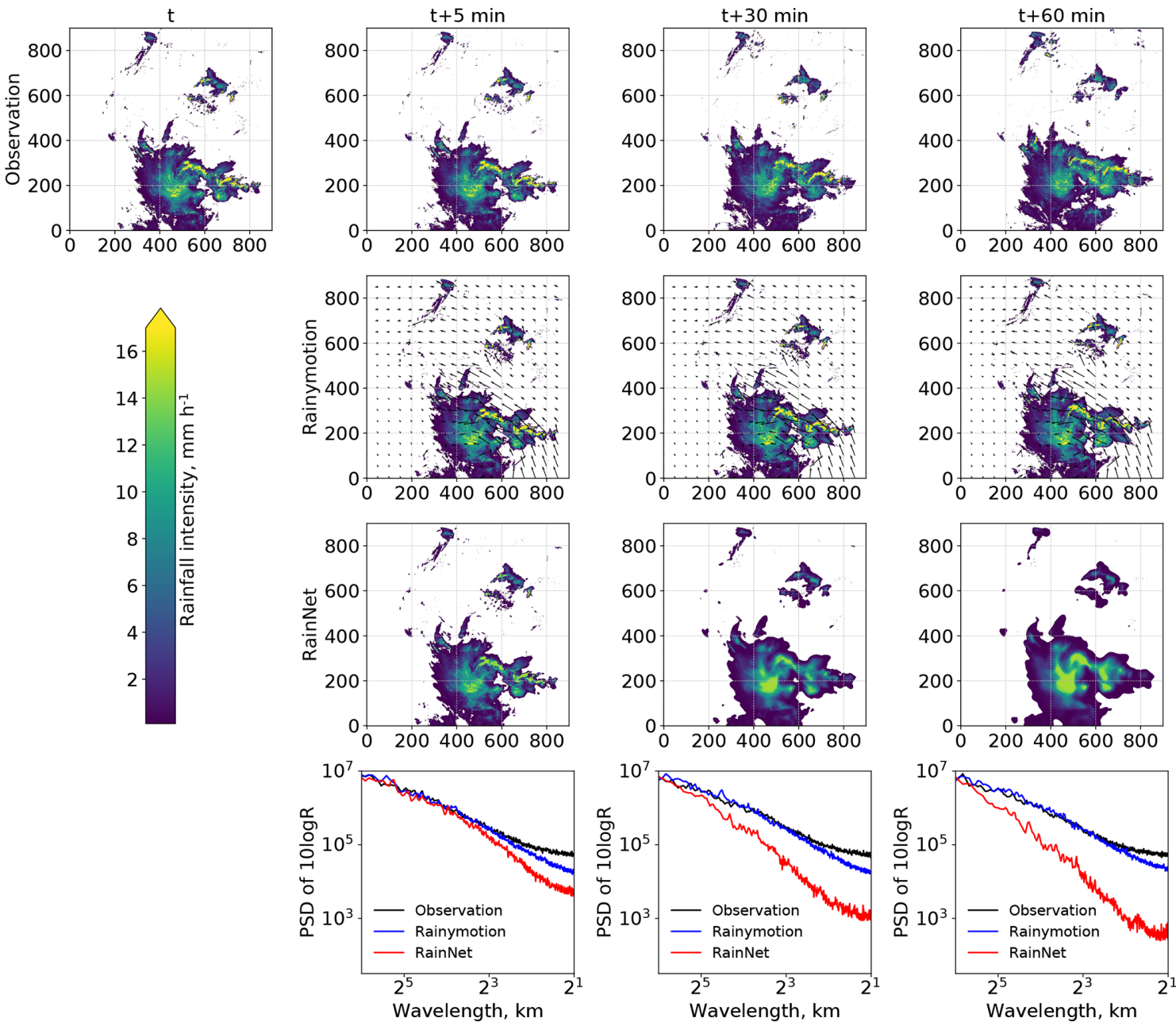

Figure 4. Precipitation observations as well as Rainymotion and RainNet nowcasts at $t=29$ May 2016, 19:15 UTC. Top row: observed precipitation intensity at time $t, t+5, t+30$, and $t+60 \mathrm{~min}$. Second row: corresponding Rainymotion predictions, together with the underlying velocity field obtained from optical flow. Bottom row: power spectral density plots for observations and nowcasts at lead times of 5, 30, and $60 \mathrm{~min}$.
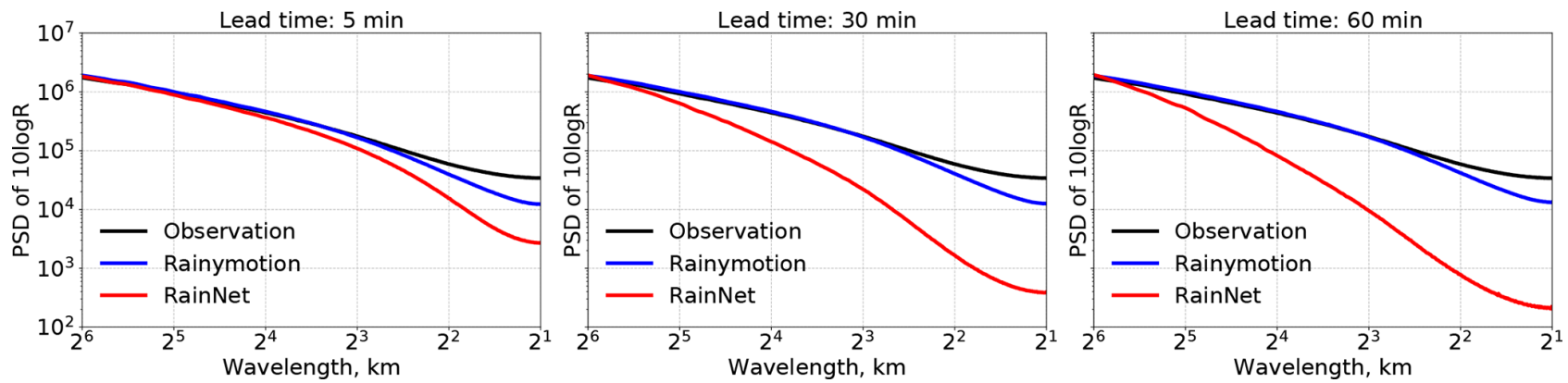

Figure 5. PSD averaged over all verification events and nowcasts for lead times of 5, 30, and $60 \mathrm{~min}$.

The dependency of the FSS (or, rather, the difference of FSS values between Rainymotion and RainNet) on spatial scale, intensity threshold, and lead time is a direct result of inherent model properties. Rainymotion advects precipitation features but preserves their intensity. When we in- crease the size of the spatial neighborhood around a pixel, this neighborhood could, at some size, include high-intensity precipitation features that Rainymotion has preserved but slightly misplaced. RainNet's loss function, however, only accounts for the native grid at $1 \mathrm{~km}$ resolution, so it has no 

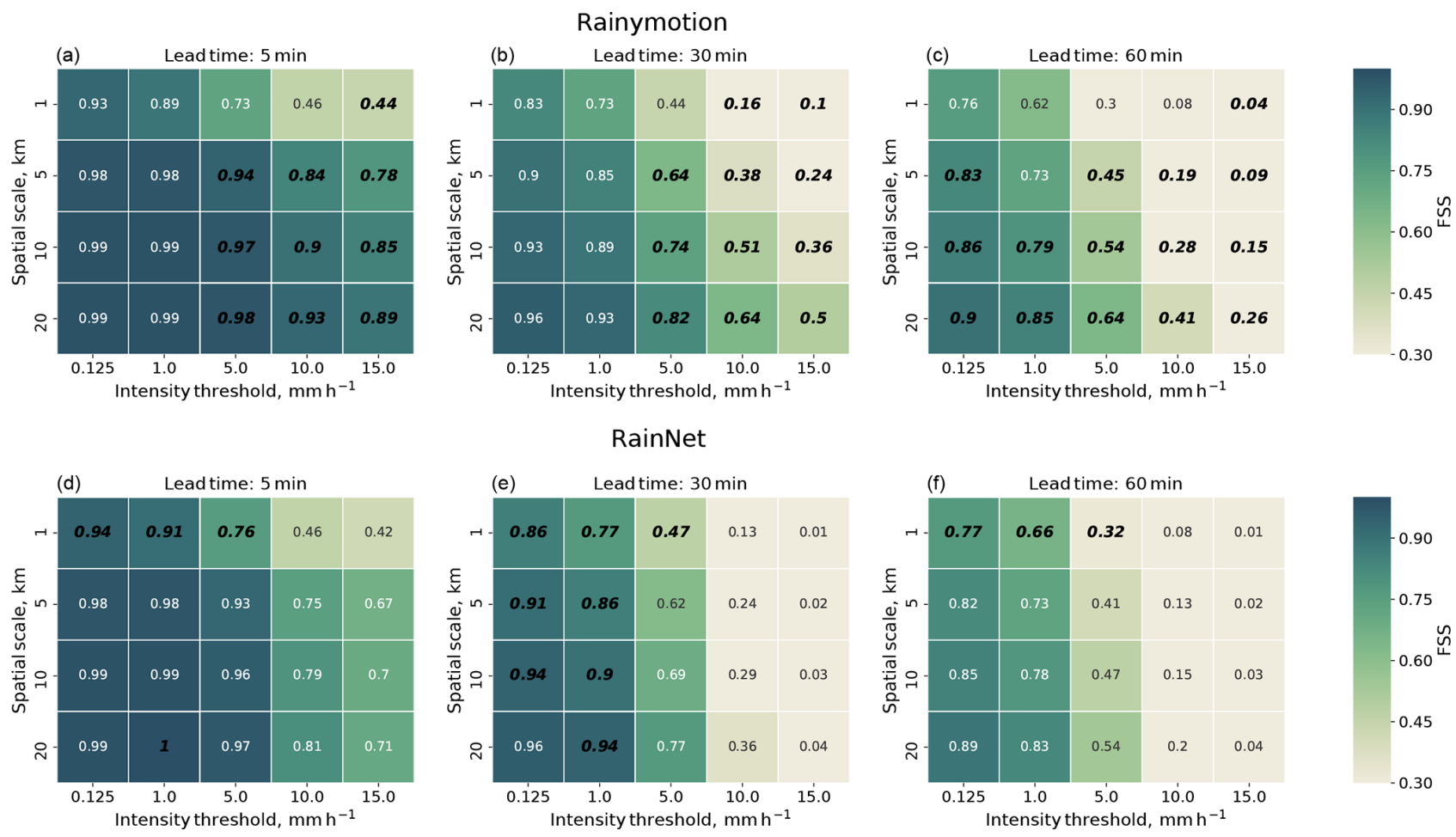

Figure 6. Fractions skill score (FSS) for Rainymotion (a, b, c) and RainNet (d, e, f) for 5, 30, and 60 min lead times, for spatial window sizes of $1,5,10$, and $20 \mathrm{~km}$, and for intensity thresholds of $0.125,1,5,10$, and $15 \mathrm{mmh}^{-1}$. In addition to the color code of the FSS, we added the numerical FSS values. The FSS values of the models which are significantly superior for a specific combination of window size, intensity threshold, and lead time are typed in bold black digits, and the inferior models are in regular digits.

notion of what could be a slight or "acceptable" displacement error. Instead, RainNet has learned spatial smoothing as an efficient way to factor in spatial uncertainty and minimize the loss function, resulting in a loss of high-intensity features. As discussed above, that effect becomes increasingly prominent for longer lead times because the effect of smoothing propagates.

\section{Summary and conclusions}

In this study, we have presented RainNet, a deep convolutional neural network architecture for radar-based precipitation nowcasting. Its design was inspired by the U-Net and SegNet families of deep learning models for binary segmentation, and it follows an encoder-decoder architecture in which the encoder progressively downscales the spatial resolution using pooling, followed by convolutional layers, and the decoder progressively upscales the learned patterns to a higher spatial resolution using upsampling, followed by convolutional layers.

RainNet was trained to predict precipitation at a lead time of $5 \mathrm{~min}$, using several years of quality-controlled weather radar composites based on the DWD weather radar network. Those data cover Germany with a spatial domain of $900 \mathrm{~km} \times 900 \mathrm{~km}$ and have a resolution of $1 \mathrm{~km}$ in space and $5 \mathrm{~min}$ in time. Independent verification experiments were carried out on 11 summer precipitation events from 2016 to 2017. In order to achieve a lead time of $60 \mathrm{~min}$, a recursive approach was implemented by using RainNet predictions at 5 min lead times as model inputs for longer lead times. In the verification experiments, Eulerian persistence served as a trivial benchmark. As an additional benchmark, we used a model from the rainymotion library which had previously been shown to outperform the operational nowcasting model of the German Weather Service for the same set of verification events.

RainNet significantly outperformed both benchmark models at all lead times up to $60 \mathrm{~min}$ for the routine verification metrics mean absolute error (MAE) and the critical success index (CSI) at intensity thresholds of $0.125,1$, and $5 \mathrm{~mm} \mathrm{~h}^{-1}$. Depending on the verification metric, these results would correspond to an extension of the effective lead time in the order of 10-20 min by RainNet as compared to Rainymotion. However, Rainymotion turned out to be clearly superior in predicting the exceedance of higher-intensity thresholds (here 10 and $15 \mathrm{~mm} \mathrm{~h}^{-1}$ ) as shown by the corresponding CSI analysis.

RainNet's limited ability to predict high rainfall intensities could be attributed to a remarkable level of spatial smoothing in its predictions. That smoothing becomes increasingly 
apparent at longer lead times. Yet it is already prominent at a lead time of $5 \mathrm{~min}$. That was confirmed by an analysis of power spectral density which showed, at time $t+5 \mathrm{~min}$, a loss of spectral power at length scales of $16 \mathrm{~km}$ and below. Obviously, RainNet has learned an optimal level of smoothing to produce a nowcast at $5 \mathrm{~min}$ lead times. In that sense, the loss of spectral power at small scales is informative as it reflects the limits of predictability as a function of spatial scale. Beyond the lead time of $5 \mathrm{~min}$, however, the increasing level of smoothing is a mere artifact - an analogue to numerical diffusion - that is not a property of RainNet itself but of its recursive application: as we repeatedly use smoothed nowcasts as model inputs, we cumulate the effect of smoothing over time. That certainly is an undesirable property, and it becomes particularly unfavorable for the prediction of high-intensity precipitation features. As was shown on the basis of the fractions skill score (FSS), Rainymotion outperforms RainNet already at an intensity of $5 \mathrm{~mm} \mathrm{~h}^{-1}$ once we start to evaluate the performance in a spatial neighborhood around the native grid pixel of $1 \mathrm{~km} \times 1 \mathrm{~km}$ size. This is because Rainymotion preserves distinct precipitation features but tends to misplace them. RainNet, however, tends to lose such features over longer lead times due to cumulative smoothing effects more so if it is applied recursively.

From an early warning perspective, that property of RainNet clearly limits its usefulness. There are, however, options to address that issue in future research.

- The loss function used in the training could be adjusted in order to penalize the loss of power at small spatial scales. The loss function explicitly represents our requirements to the model. Verifying the model by other performance metrics will typically reveal whether these metrics are rather in agreement or in conflict with these requirements. In our case, the logcosh loss function appears to favor a low MAE but at the cost of losing distinct precipitation features. In general, future users need to be aware that, apart from the network design, the optimization itself constitutes the main difference to "heuristic" tracking-and-extrapolation techniques (such as Rainymotion) which do not use any systematic parameter optimization. The training procedure will stubbornly attempt to minimize the loss function, irrespective of what researchers consider to be "physically plausible". For many researchers in the field of nowcasting, that notion might be in stark contrast to experiences with "conventional" nowcasting techniques which tend to effortlessly produce at least plausible patterns.

- RainNet should be directly trained to predict precipitation at lead times beyond $5 \mathrm{~min}$. However, preliminary training experiments with that learning task had difficulties to converge. We thus recommend to still use recursive predictions as model inputs for longer lead times during training in order to improve convergence. For example, to predict precipitation at time $t+10 \mathrm{~min}$, Rain-
Net could be trained using precipitation at time $t-15$, $t-10, \ldots, t \mathrm{~min}$ as input but using the recursive prediction at time $t+5$ as an additional input layer. While the direct prediction of precipitation at longer lead times should reduce excessive smoothing as a result of numerical diffusion, we would still expect the level of smoothing to increase with lead time as a result of the predictive uncertainty at small scales.

- As an alternative to predicting continuous values of precipitation intensity, RainNet could be trained to predict the exceedance of specific intensity thresholds instead. That would correspond to a binary segmentation task. It is possible that the objective of learning the segmentation for low intensities might be in conflict with learning it for high intensities. That is why the training could be carried out both separately and jointly for disparate thresholds in order to investigate whether there are inherent trade-offs. From an early warning perspective, it makes sense to train RainNet for binary segmentation based on user-defined thresholds that are governed by the context of risk management. The additional advantage of training RainNet to predict threshold exceedance is that we could use its output directly as a measure of uncertainty (of that exceedance).

We consider any of those options worth pursuing in order to increase the usefulness of RainNet in an early warning context - i.e., to better represent precipitation intensities that exceed hazardous thresholds. We would expect the overall architecture of RainNet to be a helpful starting point.

Yet the key issue of precipitation prediction - the anticipation of convective initialization, as well as the growth and dissipation of precipitation in the imminent future - still appears to be unresolved. It is an inherent limitation of nowcasting models purely based on optical flow: they can extrapolate motion fairly well, but they cannot predict intensity dynamics. Deep learning architectures, however, might be able to learn recurrent patterns of growth and dissipation, although it will be challenging to verify if they actually did. In the context of this study, though, we have to assume that RainNet has rather learned the representation of motion patterns instead of rainfall intensity dynamics: for a lead time of $5 \mathrm{~min}$, the effects of motion can generally be expected to dominate over the effects of intensity dynamics, which will propagate to the learning results. The fact that we actually could recursively use RainNet's predictions at 5 min lead times in order to predict precipitation at $1 \mathrm{~h}$ lead times also implies that RainNet, in essence, learned to represent motion patterns and optimal smoothing. In that case, the trained model might even be applicable on data in another region, which could be tested in future verification experiments.

Another limitation in successfully learning patterns of intensity growth and dissipation might be the input data itself. While we do not exclude the possibility that such patterns 
could be learned from just two-dimensional radar composites, other input variables might add essential information on imminent atmospheric dynamics - the predisposition of the atmosphere to produce or to dissolve precipitation. Such additional data might include three-dimensional radar volume data, dual-polarization radar moments, or the output fields of numerical weather prediction (NWP) models. Formally, the inclusion of NWP fields in a learning framework could be considered as a different way of assimilation, combining in a data-driven way - the information content of physical models and observations.

Our study provides, after Shi et al. (2015, 2017, 2018), another proof of concept that convolutional neural networks provide a firm basis to compete with conventional nowcasting models based on optical flow (most recently, Google Research has also reported similar attempts based on a U-Net architecture; see Agrawal et al., 2019). Yet this study should rather be considered as a starting point to further improve the predictive skill of convolutional neural networks and to better understand the properties of their predictions - in a statistical sense but also in how processes of motion and intensity dynamics are reflected. To that end, computational complexity and the cost of the training process still have to be considered as inhibitive, despite the tremendous progress achieved in the past years. RainNet's training would require almost a year on a standard desktop CPU in contrast to $3 \mathrm{~d}$ on a modern desktop GPU (although the latter is a challenge to implement for non-experts). Yet it is possible to run deep learning models with already optimized (pretrained) weights on a desktop computer. Thus, it is important to make available not only the code of the network architecture but also the corresponding weights, applicable using open-source software tools and libraries. We provide all this - code, pretrained weights, as well as training and verification data - as an input for future studies on open repositories (Ayzel, 2020a, b, c).

Code and data availability. The RainNet model is free and open source. It is distributed under the MIT software license which allows unrestricted use. The source code is provided through a GitHub repository https://github.com/hydrogo/rainnet (last access: 30 January 2020; Ayzel, 2020d); a snapshot of RainNet v1.0 is also available at https://doi.org/10.5281/zenodo.3631038 (Ayzel, 2020a); the pretrained RainNet model and its weights are available at https://doi.org/10.5281/zenodo.3630429 (Ayzel, 2020b). DWD provided the sample data of the RY product; it is available at https://doi.org/10.5281/zenodo.3629951 (Ayzel, 2020c).

Supplement. The supplement related to this article is available online at: https://doi.org/10.5194/gmd-13-2631-2020-supplement.

Author contributions. GA developed the RainNet model, carried out the benchmark experiments, and wrote the paper. TS and MH supervised the study and co-authored the paper.
Competing interests. The authors declare that they have no conflict of interest.

Acknowledgements. Georgy Ayzel would like to thank the Open Data Science community (https://ods.ai, last access: 10 June 2020) for many valuable discussions and educational help in the growing field of deep learning. We ran our experiments using the GPU computation resources of the Machine Learning Group of the University of Potsdam (Potsdam, Germany) and the Shared Facility Center "Data Center of FEB RAS" (Khabarovsk, Russia). We acknowledge the support of Deutsche Forschungsgemeinschaft (German Research Foundation) and the open-access publication fund of the University of Potsdam.

Financial support. This research has been supported by Geo.X, the Research Network for Geosciences in Berlin and Potsdam (grant no. SO_087_GeoX).

Review statement. This paper was edited by Simone Marras and reviewed by Scott Collis and Gabriele Franch.

\section{References}

Agrawal, S., Barrington, L., Bromberg, C., Burge, J., Gazen, C., and Hickey, J.: Machine Learning for Precipitation Nowcasting from Radar Images, available at: https://arxiv.org/abs/1912.12132 (last access: 28 January 2020), 2019.

Austin, G. L. and Bellon, A.: The use of digital weather radar records for short-term precipitation forecasting, Q. J. Roy. Meteor. Soc., 100, 658-664, https://doi.org/10.1002/qj.49710042612, 1974.

Ayzel, G.: hydrogo/rainnet: RainNet v1.0-gmdd, Zenodo, https://doi.org/10.5281/zenodo.3631038, 2020a.

Ayzel, G.: RainNet: pretrained model and weights, Zenodo, https://doi.org/10.5281/zenodo.3630429, 2020b.

Ayzel, G.: RYDL: the sample data of the RY product for deep learning applications, Zenodo, https://doi.org/10.5281/zenodo.3629951, 2020c.

Ayzel, G.: RainNet: a convolutional neural network for radarbased precipitation nowcasting, available at: https://github.com/ hydrogo/rainnet, last access: 10 June 2020.

Ayzel, G., Heistermann, M., and Winterrath, T.: Optical flow models as an open benchmark for radar-based precipitation nowcasting (rainymotion v0.1), Geosci. Model Dev., 12, 1387-1402, https://doi.org/10.5194/gmd-12-1387-2019, 2019.

Badrinarayanan, V., Kendall, A., and Cipolla, R.: SegNet: A Deep Convolutional Encoder-Decoder Architecture for Image Segmentation, IEEE T. Pattern Anal., 39, 2481-2495, https://doi.org/10.1109/TPAMI.2016.2644615, 2017.

Bai, S., Kolter, J. Z., and Koltun, V.: An Empirical Evaluation of Generic Convolutional and Recurrent Networks for Sequence Modeling, available at: https://arxiv.org/abs/1803.01271 (last access: 28 January 2020), 2018. 
Bauer, P., Thorpe, A., and Brunet, G.: The quiet revolution of numerical weather prediction, Nature, 525, 47-55, https://doi.org/10.1038/nature14956, 2015.

Boureau, Y.-L., Ponce, J., and LeCun, Y.: A Theoretical Analysis of Feature Pooling in Visual Recognition, in: Proceedings of the 27th International Conference on International Conference on Machine Learning, ICML'10, Omnipress, Madison, WI, USA, 21-24 June 2010, Haifa, Israel, 111-118, 2010.

Chen, P., Chen, G., and Zhang, S.: Log Hyperbolic Cosine Loss Improves Variational Auto-Encoder, available at: https:// openreview.net/forum?id=rkglvsC9Ym (last access: 28 January 2020), 2018.

Chollet, F. et al.: Keras, https://keras.io (last access: 10 June 2020), 2015.

Dahl, G. E., Sainath, T. N., and Hinton, G. E.: Improving deep neural networks for LVCSR using rectified linear units and dropout, in: 2013 IEEE International Conference on Acoustics, Speech and Signal Processing, 26-31 May 2013, Vancouver, Canada, 8609-8613, https://doi.org/10.1109/ICASSP.2013.6639346, 2013.

Dueben, P. D. and Bauer, P.: Challenges and design choices for global weather and climate models based on machine learning, Geosci. Model Dev., 11, 3999-4009, https://doi.org/10.5194/gmd-11-3999-2018, 2018.

Gehring, J., Auli, M., Grangier, D., Yarats, D., and Dauphin, Y. N.: Convolutional Sequence to Sequence Learning, in: Proceedings of the 34th International Conference on Machine Learning - Volume 70, ICML'17, 6-11 August 2017,Sydney, Australia, 12431252, JMLR.org, 2017.

Gentine, P., Pritchard, M., Rasp, S., Reinaudi, G., and Yacalis, G.: Could Machine Learning Break the Convection Parameterization Deadlock?, Geophys. Res. Lett., 45, 5742-5751, https://doi.org/10.1029/2018GL078202, 2018.

Germann, U. and Zawadzki, I.: Scale-Dependence of the Predictability of Precipitation from Continental Radar Images. Part I: Description of the Methodology, Mon. Weather Rev., 130, 2859-2873, https://doi.org/10.1175/15200493(2002)130<2859:SDOTPO>2.0.CO;2, 2002.

Gu, J., Wang, Z., Kuen, J., Ma, L., Shahroudy, A., Shuai, B., Liu, T., Wang, X., Wang, G., Cai, J., and Chen, T.: Recent advances in convolutional neural networks, Pattern Recogn., 77, 354-377, https://doi.org/10.1016/j.patcog.2017.10.013, 2018.

Iglovikov, V. and Shvets, A.: TernausNet: U-Net with VGG11 Encoder Pre-Trained on ImageNet for Image Segmentation, available at: https://arxiv.org/abs/1801.05746 (last access: 28 January 2020), 2018.

Kingma, D. P. and Ba, J.: Adam: A Method for Stochastic Optimization, in: 3rd International Conference on Learning Representations, ICLR 2015, San Diego, CA, USA, 7-9 May 2015, Conference Track Proceedings, edited by: Bengio, Y. and LeCun, Y., available at: http://arxiv.org/abs/1412.6980 (last access: 10 June 2020), 2015.

Krizhevsky, A., Sutskever, I., and Hinton, G. E.: ImageNet Classification with Deep Convolutional Neural Networks, in: Advances in Neural Information Processing Systems 25, NIPS 2012, Lake Tahoe, Nevada, USA, 3-9 December 2012, Curran Associates, Inc. Red Hook, NY, USA, edited by: Pereira, F., Burges, C. J. C., Bottou, L., and Weinberger, K. Q., 1097-1105, available at: http://papers.nips.cc/paper/ 4824-imagenet-classification-with-deep-convolutional-neural-networks. pdf (last access: 10 June 2020), 2012.

LeCun, Y., Bengio, Y., and Hinton, G.: Deep learning, Nature, 521, 436-444, https://doi.org/10.1038/nature14539, 2015.

Lin, C., Vasić, S., Kilambi, A., Turner, B., and Zawadzki, I.: Precipitation forecast skill of numerical weather prediction models and radar nowcasts, Geophys. Res. Lett., 32, L14801, https://doi.org/10.1029/2005GL023451, 2005.

Long, J., Shelhamer, E., and Darrell, T.: Fully Convolutional Networks for Semantic Segmentation, in: The IEEE Conference on Computer Vision and Pattern Recognition (CVPR), 8-12 June 2015, Boston, Massachusetts, USA, 2015.

Mittermaier, M. and Roberts, N.: Intercomparison of Spatial Forecast Verification Methods: Identifying Skillful Spatial Scales Using the Fractions Skill Score, Weather Forecast., 25, 343-354, https://doi.org/10.1175/2009WAF2222260.1, 2010.

Nair, V. and Hinton, G. E.: Interpersonal Informatics: Making Social Influence Visible, in: Proceedings of the 27th International Conference on International Conference on Machine Learning, ICML'10, Omnipress, Madison, WI, USA, 21-24 June 2010, Haifa, Israel, 807-814, 2010.

Reichstein, M., Camps-Valls, G., Stevens, B., Jung, M., Denzler, J., Carvalhais, N., and Prabhat: Deep learning and process understanding for data-driven Earth system science, Nature, 566, 195204, https://doi.org/10.1038/s41586-019-0912-1, 2019.

Reyniers, M.: Quantitative precipitation forecasts based on radar observations: Principles, algorithms and operational systems, Institut Royal Météorologique de Belgique, available at: https://www.meteo.be/meteo/download/fr/3040165/pdf/rmi_ scpub-1261.pdf (last access: 10 June 2020), 2008.

Ronneberger, O., Fischer, P., and Brox, T.: U-Net: Convolutional Networks for Biomedical Image Segmentation, in: Medical Image Computing and Computer-Assisted Intervention - MICCAI 2015, edited by: Navab, N., Hornegger, J., Wells, W. M., and Frangi, A. F., Springer International Publishing, Cham, pp. 234241, https://doi.org/10.1007/978-3-319-24574-4_28, 2015.

Shi, E., Li, Q., Gu, D., and Zhao, Z.: A Method of Weather Radar Echo Extrapolation Based on Convolutional Neural Networks, in: MultiMedia Modeling, edited by: Schoeffmann, K., Chalidabhongse, T. H., Ngo, C. W., Aramvith, S., O'Connor, N. E., Ho, Y.-S., Gabbouj, M., and Elgammal, A., Springer International Publishing, Cham, pp. 16-28, https://doi.org/10.1007/9783-319-73603-7_2, 2018.

Shi, X., Chen, Z., Wang, H., Yeung, D.-Y., Wong, W.-k., and Woo, W.-c.: Convolutional LSTM Network: A Machine Learning Approach for Precipitation Nowcasting, in: Advances in Neural Information Processing Systems 28, edited by: Cortes, C., Lawrence, N. D., Lee, D. D., Sugiyama, M., and Garnett, R., Curran Associates, Inc., Red Hook, NY, USA, 802-810, available at: http://papers.nips.cc/paper/5955-convolutional-lstm-networka-machine-learning-approach-for-precipitation-nowcasting.pdf (last access: 10 June 2020), 2015.

Shi, X., Gao, Z., Lausen, L., Wang, H., Yeung, D.-Y., Wong, W.-k., and Woo, W.-c.: Deep Learning for Precipitation Nowcasting: A Benchmark and A New Model, in: Advances in Neural Information Processing Systems 30, edited by: Guyon, I., Luxburg, U. V., Bengio, S., Wallach, H., Fergus, R., Vishwanathan, S., and Garnett, R., Curran Associates, Inc., Red Hook, NY, USA, 5617-5627, available at: http://papers.nips.cc/paper/7145-deep- 
learning-for-precipitation-nowcasting-a-benchmark-and-a-newmodel.pdf (last access: 10 June 2020), 2017.

Singh, S., Sarkar, S., and Mitra, P.: Leveraging Convolutions in Recurrent Neural Networks for Doppler Weather Radar Echo Prediction, in: Advances in Neural Networks - ISNN 2017, edited by: Cong, F., Leung, A., and Wei, Q., Springer International Publishing, Cham, pp. 310-317, https://doi.org/10.1007/978-3-31959081-3_37, 2017.

Srivastava, N., Hinton, G., Krizhevsky, A., Sutskever, I., and Salakhutdinov, R.: Dropout: A Simple Way to Prevent Neural Networks from Overfitting, J. Mach. Learn. Res., 15, 1929-1958, 2014.

Srivastava, R. K., Greff, K., and Schmidhuber, J.: Training Very Deep Networks, in: Advances in Neural Information Processing Systems 28, edited by: Cortes, C., Lawrence, N. D., Lee, D. D., Sugiyama, M., and Garnett, R., Curran Associates, Inc., Red Hook, NY, USA, 2377-2385, available at: http://papers.nips. cc/paper/5850-training-very-deep-networks.pdf (last access: 10 June 2020), 2015.

Sun, J., Xue, M., Wilson, J. W., Zawadzki, I., Ballard, S. P., OnvleeHooimeyer, J., Joe, P., Barker, D. M., Li, P.-W., Golding, B., $\mathrm{Xu}$, M., and Pinto, J.: Use of NWP for Nowcasting Convective Precipitation: Recent Progress and Challenges, B. Am. Meteorol. Soc., 95, 409-426, https://doi.org/10.1175/BAMS-D-1100263.1, 2014.
Sutskever, I., Vinyals, O., and Le, Q. V.: Sequence to Sequence Learning with Neural Networks, in: Advances in Neural Information Processing Systems 27, edited by: Ghahramani, Z., Welling, M., Cortes, C., Lawrence, N. D., and Weinberger, K. Q., Curran Associates, Inc., Red Hook, NY, USA, 3104-3112, available at: http://papers.nips.cc/paper/ 5346-sequence-to-sequence-learning-with-neural-networks.pdf (last access: 10 June 2020), 2014.

Welch, P.: The use of fast Fourier transform for the estimation of power spectra: a method based on time averaging over short, modified periodograms, IEEE T. Audio Electroacoust., 15, 7073, 1967.

Wilson, J. W., Crook, N. A., Mueller, C. K., Sun, J., and Dixon, M.: Nowcasting Thunderstorms: A Status Report, B. Am. Meteorol. Soc., 79, 2079-2099, https://doi.org/10.1175/15200477(1998)079<2079:NTASR>2.0.CO;2, 1998. 